\title{
Interaction of Flavin-Dependent Fructose Dehydrogenase with Cytochrome $c$ as Basis for the Construction of Biomacromolecular Architectures on Electrodes
}

\author{
Christoph Wettstein ${ }^{\ddagger}$, Kenji Kano ${ }^{\dagger}$, Daniel Schäfert, Ulla Wollenberger ${ }^{\S}$, Fred Lisdat ${ }^{\ddagger}, *$ \\ ${ }^{*}$ Technical University of Applied Sciences Wildau, Institute of Applied Life Sciences, \\ Biosystems Technology, Hochschulring 1, 15745 Wildau, Germany \\ ${ }^{\dagger}$ Division of Applied Life Sciences, Graduate School of Agriculture, Kyoto University, Sakyo, \\ Kyoto 606-8502, Japan \\ ${ }^{\S}$ Institute of Biochemistry and Biology, University Potsdam, Karl-Liebknecht-Str. 24-25, 14476 \\ Potsdam/ Golm, Germany
}

Corresponding Author

*Email for Fred Lisdat: fred.lisdat@th-wildau.de

\section{TABLE OF CONTENS}

S1 .........Cyclic voltammograms of cyt $c$ monolayer electrode with fructose and FDH in solution S2 .........Cyclic voltammograms of cyt $\mathrm{c}$ monolayer electrode with Triton X-100

S3 .........Cyclic voltammograms of cyt c monolayer electrode at $\mathrm{pH} 4.5$

S4.........Cyclic voltammograms of cyt $c$ monolayer electrode with adsorbed of FDH S5.........Cyclic voltammograms of cyt $c$-FDH electrode before and after addition of fructose 
Cyclic voltammograms of a cyt $c$ monolayer electrode with fructose (FR) in solution and in the presence of different FDH concentrations.

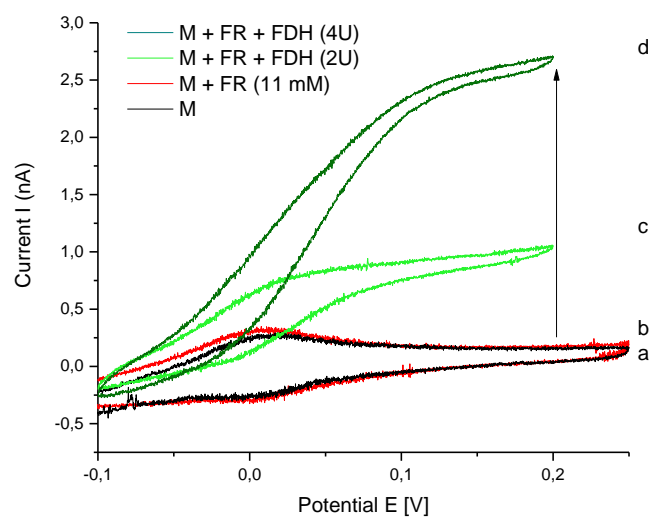

Figure S1: Cyclic voltammograms of a cyt $c$ monolayer (M) on a MU:MUA modified AuE surface with $11 \mathrm{mM}$ FR in solution. Monolayer (1), M with FR in solution (2), in presence of $2 \mathrm{U} \mathrm{FDH} \mathrm{(3)} \mathrm{and} 4 \mathrm{U}$ FDH (4). Experimental conditions: $5 \mathrm{mM} \mathrm{KPi}, \mathrm{pH} 7.0, \mathrm{SR}=2 \mathrm{mV} / \mathrm{s}$. 
Cyclic voltammograms of a cyt c monolayer electrode with FDH in solution and in the presence and the absence of Triton X-100 (TX).

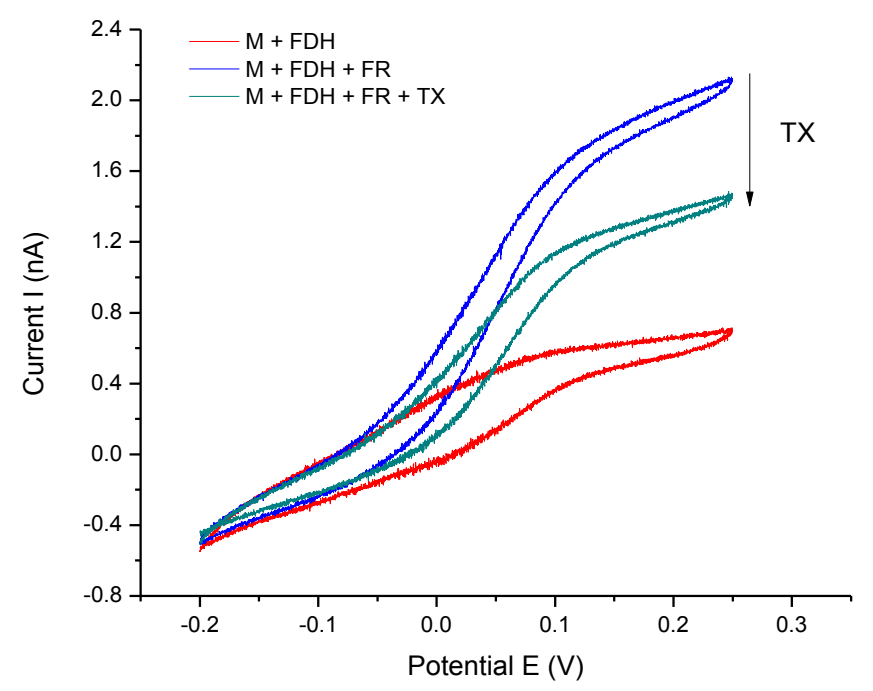

Figure S2: Cyclic voltammograms of a cyt $c$ monolayer (M) on a MU:MUA modified AuE surface with $4 \mathrm{U}$ FDH in solution. $\mathrm{M}$ with FDH in solution (red line), in presence of $44.6 \mathrm{mM}$ FR (blue line) and upon addition $0.1 \% \mathrm{TX}$ (green line). Experimental conditions: $5 \mathrm{mM} \mathrm{KPi}$, $\mathrm{pH} 7.0, \mathrm{SR}=2 \mathrm{mV} / \mathrm{s}$.

It has been reported that FDH is inactivated by precipitation in the absence of stabilizing detergents (Ameyama, et al., 1981). Therefore, control experiments were performed in $0.1 \%$ Triton X-100 (TX) containing buffer (figure S2). With respect to the results obtained from TXfree solutions (blue line), a decrease in the catalytic activity was found after the addition of the detergent (green line). The cyt $c$ electrochemistry does not seem to be affected by TX. However, the electron transfer (ET) reaction between cyt $c$ and FDH may be hindered by the detergent. 
Although it was reported that FDH is inactivated in the absence of TX (Ameyama, et al., 1981), the enzyme appears to remain active in TX-free buffer, at least in the time range of the measurements. In order to create favourable conditions for the investigation of inter-protein ET reactions and also to minimize the risk of an interference of the detergent with the test system, all electrochemical measurements were performed in TX-free buffer. 
Cyclic voltammograms of surface bound cyt $c$ with freely diffusing FDH at $\mathrm{pH} 4.5$.

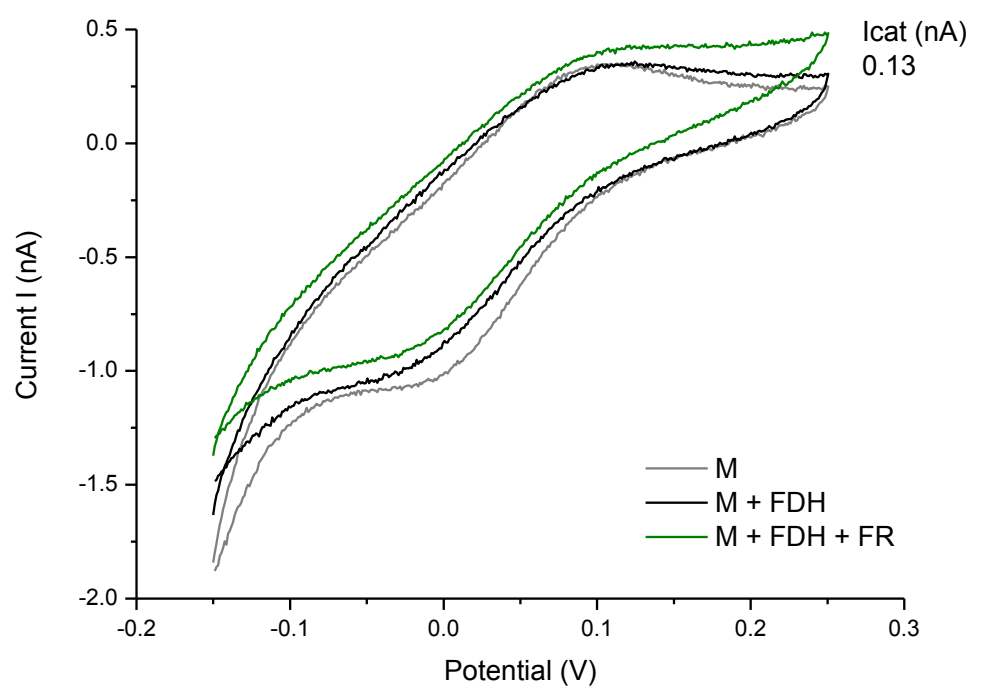

Figure S3: Cyclic voltammograms of a cyt $c$ monolayer (M) on a MU:MUA modified AuE surface $(20 \mathrm{mM} \mathrm{KPi}, \mathrm{SR}=2 \mathrm{mV} / \mathrm{s})$ at $\mathrm{pH} 4.5 . \mathrm{M}$ (grey line), $\mathrm{M}$ with FDH (4 $\mathrm{U})$ in solution (black line) and in presence of $44.6 \mathrm{mM}$ fructose (green line). 
Cyclic voltammograms of a cyt $c$ monolayer electrode after direct adsorption of FDH.

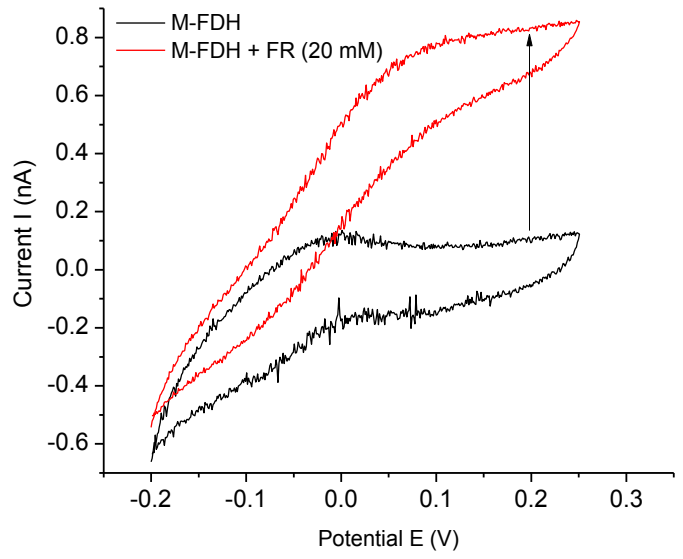

Figure S4: Cyclic voltammograms of a cyt $c$-FDH electrode (AuE/MU:MUA/cyt $c$-FDH) at pH 7.0 (5 mM KPi, SR $=2 \mathrm{mV} / \mathrm{s})$. Monolayer (M) with FDH immobilized on top (black line) and upon addition of $20 \mathrm{mM}$ fructose (red line). 
Cyclic voltammograms of a cyt $c$-FDH electrode, at $100 \mathrm{mV} / \mathrm{s}$, before and after study of fructose-induced bioelectrocatalysis.

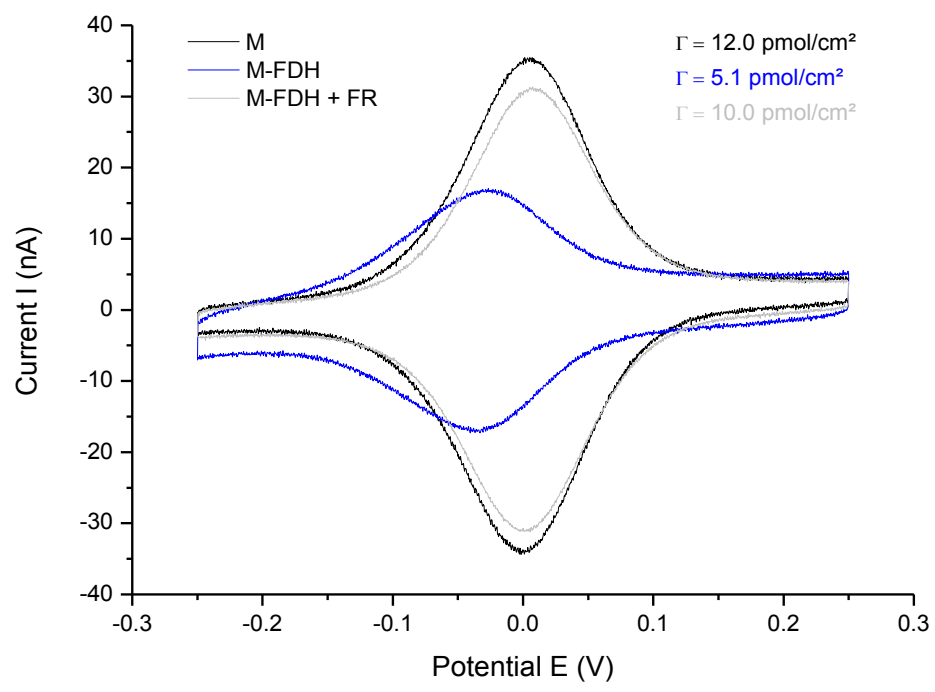

Figure S5: Cyclic voltammograms of a cyt $c$-FDH electrode (AuE/MU:MUA/cyt $c$-FDH, FDH adsorbed onto the cyt $c$ monolayer electrode) at $\mathrm{pH} 7.0(5 \mathrm{mM} \mathrm{KPi}, \mathrm{SR}=100 \mathrm{mV} / \mathrm{s})$. Monolayer (M) (black line), M with FDH adsorbed on top (blue line) and M with FDH adsorbed on top after the measurement in fructose containing solution at small scan rate (grey line).

The cyt $c$ signal is influenced by the FDH adsorption. The recovery of the cyt $c$ signal indicates that cyt $c$ is not removed by the FDH binding, but FDH is not so tightly bound to the cyt $c$ monolayer so that it can desorb from the electrode particularly in fructose containing solution. The cyt $c$ in contrast is rather stably bound to the electrode. 